\title{
Will Genotyping Replace Serology in Future Routine Blood Grouping? - Opinion 4
}

\section{Personalized versus Universal Blood Transfusions - Combining the Efforts}

\author{
Jill R. Storry Martin L. Olsson
}

Division of Hematology and Transfusion Medicine, Department of Laboratory Medicine, Lund University \& Blood Centre, Lund University Hospital, Sweden

The core question of this forum addresses whether genotyping will replace serology in future routine blood grouping. Our response to that question is another: will genotyping need to replace serology? The past two decades in transfusion medicine have witnessed development in two different directions: the older path is one of developing a 'universal' blood supply where all blood is somehow converted to an inert group-O-like status. The Holy Grail is to eliminate pretransfusion testing, permit the transfusion of any unit of blood to any person and to prevent possible post-transfusion $\mathrm{ABO}$ related haemolytic events. The more recent path leads in the opposite direction, i.e. development of more comprehensive genotyping platforms so that patient and blood donor may be matched specifically for as many antigens as possible and thus limit allo-immunisation events through antigen compatibility. This mimics our current testing practices although on a more grandiose scale and answers the primary forum question with a probable yes, since discrimination at the molecular level is much more comprehensive than that which can be achieved serologically.

The bases of the high-throughput genotyping platforms are discussed in detail elsewhere in this journal; different approaches to developing universal blood have also been the subject of comprehensive investigation. It is not our intention to review them again but more to look objectively at the value of the new developments and see if indeed they have a role in improving the way we provide blood to patients.

Personalised medicine is a continuously expanding field based on the knowledge of the inter-individual variations in the human genome and increased understanding of the molecular basis of disease. Identification of molecular markers as risk factors for allo-immunisation would provide means to select patients who could benefit the most from more wellmatched red cell transfusions and constitute a more prophylactic approach than today's.
Current practice is to match patients and donors for ABO and $\mathrm{RhD}$ antigens at a minimum. Matching for $\mathrm{K}$ and $\mathrm{c}$ antigens in female patients of child-bearing age is also common in many transfusion services. Discovery of the molecular bases behind most of the clinically important blood group antigens has prompted the development of genotyping platforms on the premise that if blood for transfusion is more closely matched to the genotype of the patient, then allo-immunisation events can be decreased or possibly even avoided. These events occur in an estimated 1-2\% of transfusions and may delay medical care, prolong hospital stay, and result in less than optimal medical treatment. After a reasonable dose of scepticism initially, many blood centres and transfusion services are now competing to install systems able to achieve this goal. However, cost-efficiency is still a central issue.

There have been two primary pathways in the approach to universal blood: one has been to mask the erythrocyte surface antigens using polyethylene glycol (PEG) and its derivatives; the other has been to enzymatically cleave the A and B antigens, which are the single greatest barrier in transfusion medicine. PEG technology, which looked very promising initially, has proven to be more immunogenic than anticipated. Furthermore, masking of $\mathrm{A}$ and $\mathrm{B}$ antigens has also proven difficult to accomplish at moderate doses of PEG. These results are echoed by enzymatic removal strategies, which appear to show promise, but still transfusion of red cells against anti-A and anti-A,B following removal of A antigens remains to be proven clinically viable. However, proof of principle has been achieved for transfusion of treated group B red cells into group $\mathrm{O}$ or $\mathrm{A}$ healthy volunteer and patient recipients. A third more recent approach to the production of a universal blood supply is the large-scale in vitro culture of erythrocytes in bioreactors. The exciting potential of this technology is that one could potentially knock out genes for blood group antigens that were not required for the function of the erythrocyte. In

\section{KARGER}

Fax +497614520714

Information@Karger.de

www.karger.com (c) 2009 S. Karger GmbH, Freiburg

Accessible online at:

www.karger.com/tmh
Prof. Dr. med. Martin L. Olsson

Division of Hematology and Transfusion Medicine, Department of Laboratory Medicine

Lund University \& Blood Centre, Lund University Hospital

22185 Lund, Sweden

Martin_L.Olsson@med.lu.se 
any case, the phenotype could be selected so as to provide the most immunogenically inert form. The current major challenges in this field are production costs and the realisation of mass production.

So, how do these different approaches help in answering the question posed by this forum? The single greatest blood group-related danger to transfusion recipients remains the ABO system. Unfortunately, the complexity of the $A B O$ genes when compared with the relative simplicity of serological typing and availability of inexpensive reagents, is such that it is unlikely that anyone would rely on genotyping only for the assignation of $\mathrm{ABO}$ group to a unit of blood. However, as part of a blood group microarray the genotyping information obtained could add to the value of routine serology, e.g. in identification of weak A or B subgroups or low-grade chimeras missed by serological routine tests.

But what about the other blood group systems? There certainly, genotyping could replace serological testing. Typing for $\mathrm{RhD}$ remains imperfect serologically and is dependent on the clone(s) of anti-D used and also the number and specificity of $\mathrm{D}$ epitopes carried by the red cells. The molecular basis of $\mathrm{RhD}$ variants are readily incorporated into a genotyping approach and have been shown in one trial with BLOODchip $^{\circledR}$ (Progenika Biopharma, Derio, Spain) to correlate well with serological expression. What about new alleles that have the potential to result in an erroneous prediction of phenotype? As always, the discussion is completely different for patients/recipients and donors. If a patient is predicted to be $\mathrm{RhD}$-positive despite really being $\mathrm{RhD}$-negative, e.g. due to technical error or an unidentified pseudogene, it may lead to $\mathrm{RhD}$ immunisation. Thus, for patient typing the same precautionary principle discussed above for $A B O$ genotyping may apply. But what about a mistyped unit, e.g. predicted RhDpositive due to a pseudogene when it really is $\mathrm{RhD}$-negative? These will always exist but is it best use of medical testing re- sources to ensure that each and every one is captured? For other blood group antigens of clinical importance, genotyping offers another dimension and an added value beyond today's standard of routine care. Therefore, rare errors, most of which will probably be false-positive predictions, could be more acceptable since the advantages outnumber the risks. The major remaining question again is cost. Should this practice be used for all donors, or with whom should we start? It is quite likely that blood centres need a start-up phase anyway so stable group $\mathrm{O}$ donors are probably a cost-efficient group to start with. Subsequently, all donors but also all non-acute patients could or should be typed in order to decrease alloimmunisation, based on a priority matrix selecting the optimal unit for each patient at any given time. While the result of a donor typing can be recycled and utilised throughout the donor's life, patient typing should perhaps initially be limited to chronically transfused recipients. Again, for the full benefit of mass-scale genotyping efforts also other patient categories should be considered.

Perhaps a combined approach using enzymatic conversion (ECO) to remove A and B antigens together with a genotyping platform for all other clinically relevant antigens will enable us to obtain an optimised blood supply eventually. In addition, the array could help assure that the ABO groups were correctly assigned for selection of A- or B-degrading exoglycosidase for conversion. The advantages are clear: in addition to eliminating ABO-associated haemolytic events, the availability of ECO blood would improve inventory management and other supply chain issues since all blood will be available to any patient independent of $\mathrm{ABO}$ group, perhaps most importantly in emergency situations or massive bleeding. Computerized genotype information for all other blood group systems will decrease the risk of clerical or serological errors and ensure that the best possible match for a patient can be made each time blood is required. 